EPJ Web of Conferences 49, 12011 (2013)

DOI: 10.1051/epjconf/20134912011

(C) Owned by the authors, published by EDP Sciences, 2013

\title{
The search for the Standard Model Higgs boson produced in association with a vector boson and decaying to bottom quarks with the ATLAS detector
}

\author{
P.J. Clark on behalf of the ATLAS collaboration ${ }^{1, a}$ \\ ${ }^{1}$ SUPA - School of Physics and Astronomy, University of Edinburgh, Edinburgh, United Kingdom
}

\begin{abstract}
These proceedings present an updated search for the Standard Model Higgs boson produced in association with a $W$ or $Z$ boson and decaying to $b \bar{b}$. The analysis uses $4.7 \mathrm{fb}^{-1}$ of LHC proton-proton collision data at $\sqrt{s}=7 \mathrm{TeV}$ and $13.0 \mathrm{fb}^{-1}$ at $\sqrt{s}=8 \mathrm{TeV}$ recorded by the ATLAS experiment. The search is performed using events containing zero, one or two electrons or muons targeting the decay modes $Z H \rightarrow v \bar{v} b \bar{b}, W H \rightarrow$ $\ell v b \bar{b}$ and $Z H \rightarrow \ell^{+} \ell^{-} b \bar{b}$. No significant excess is observed. For $m_{H}=125 \mathrm{GeV}$, the observed (expected) upper 95\% confidence level limit on the cross section times the branching ratio is 1.8 (1.9) times the Standard Model prediction. A Standard Model Higgs boson with $m_{H}=110 \mathrm{GeV}$ is excluded. As a validation of the analysis method, the production of diboson pairs, $W Z$ and $Z Z$, with a $Z$ boson decaying to $b \bar{b}$, has been observed with a significance of 4.0 standard deviations and a rate compatible with the Standard Model expectation. A search was also made in the $t \bar{t} H$ channel using $4.7 \mathrm{fb}^{-1}$ of LHC data at $\sqrt{s}=7 \mathrm{TeV}$, focusing on the semileptonic decay mode of the $t \bar{t}$ system. For a Higgs boson with $m_{H}=125 \mathrm{GeV}$, the observed (expected) upper 95\% confidence level limit is 13.1 (10.5) times the Standard Model using $t \bar{t} H$.
\end{abstract}

\section{Introduction}

The search for the Standard Model (SM) Higgs Boson [14] has been one of the highest priorities in the field of particle physics. The observation of a boson decaying to a pair of photons or massive vector bosons has been recently reported by the ATLAS [5] and CMS [6] collaborations. To test whether it is the SM Higgs boson, it is important to confirm that it decays into fermion pairs. The decay to $b$ quarks is important since this the dominant decay mode in the $\mathrm{SM}$ at this mass $(B R(H \rightarrow b \bar{b}) \approx 58 \%$ [7] $)$. Measurements by the experiments at the Tevatron have reported an excess of events in the $b \bar{b}$ decay mode corresponding to about three standard deviations [8]. The CMS collaboration has set expected and observed upper limits on the production cross section times branching ratio of 1.6 and 2.1 times the Standard Model expectation, respectively [6].

These proceedings contain an update [9] of the results reported in Ref. [10] on the search for the Higgs boson decaying to a pair of $b$-quarks, produced in association with a $W$ or $Z$ boson (jointly denoted as $V$ ), giving the final states $Z H \rightarrow v \bar{v} b \bar{b}, W H \rightarrow \ell v b \bar{b}$ and $Z H \rightarrow \ell^{+} \ell^{-} b \bar{b}$, where $\ell$ refers to either an electron or a muon. The cross section for associated production with $W$ or $Z$ bosons is more than an order of magnitude lower than that of the dominant gluon fusion process, but the decay signature is much cleaner. The datasets considered here are $4.7 \mathrm{fb}^{-1}$ at $\sqrt{s}=7 \mathrm{TeV}$ delivered by the LHC in 2011 and $13.0 \mathrm{fb}^{-1}$ at $\sqrt{s}=8 \mathrm{TeV}$ delivered by the LHC in 2012. The analysis is performed for events containing zero, one or two electrons

\footnotetext{
a e-mail: P.J.Clark@ed.ac.uk
}

or muons, targeting the three Higgs boson decay channels mentioned above. The 0-lepton channel is most sensitive to $Z H$ production, with a small although not insignificant contribution from $W H$ production. The sensitivity of the 1-lepton channel is dominated by $W H$ production with a small contribution from $Z H$, whereas the 2-lepton channel is only sensitive to $Z H$ production. The channels are split into further categories, depending on the vector boson transverse momentum, and the number of jets.

There have been several improvements to the analysis to improve the sensitivity since the previous publication [10]. The most important improvements are a reoptimisation of the topological selection criteria for all three channels, increased lepton identification efficiency, the addition of further categories with different signal to background ratios, and improvements to the di- $b$-jet invariant mass resolution.

A search has also been made in the $t \bar{t} H$ channel, which is not discussed here due to space limitations. The reader is referred to Ref. [11]

\section{The ATLAS detector}

The ATLAS detector [12] consists of an inner tracker immersed in a $2 \mathrm{~T}$ magnetic field produced by a superconducting solenoid. Charged particle position and momentum measurements are made by silicon detectors in the pseudorapidity range $|\eta|<2.5$ and straw tubes in the range $|\eta|<2.0$. This is surrounded by lead liquid-argon $(0<|\eta|<3.2)$ and copper liquid argon $(3.1<|\eta|<4.9)$ 
electromagnetic calorimeters. These are enclosed by central (forward) steel scintillating-tile (copper and tungsten liquid-argon) hadronic calorimeters. The muon spectrometer comprises three large toroidal magnets, each containing eight superconducting coils, and a system of precision tracking detectors.

\section{Event selection}

The event selection is summarised in Tables 1 and 2 . Events containing zero leptons are split into six categories depending on whether they contain two or three jets and on the missing transverse energy: $120<E_{\mathrm{T}}^{\mathrm{miss}} \leq 160 \mathrm{GeV}$, $160<E_{\mathrm{T}}^{\text {miss }} \leq 200 \mathrm{GeV}$ and $E_{\mathrm{T}}^{\text {miss }}>200 \mathrm{GeV}$. Events containing one or two leptons are divided into five categories of transverse momentum of the vector boson, $p_{\mathrm{T}}^{V}$ : $p_{\mathrm{T}}^{V} \leq 50 \mathrm{GeV}, 50<p_{\mathrm{T}}^{V} \leq 100 \mathrm{GeV}, 100<p_{\mathrm{T}}^{V} \leq 150 \mathrm{GeV}$, $150<p_{\mathrm{T}}^{V} \leq 200 \mathrm{GeV}$ and $p_{\mathrm{T}}^{V}>200 \mathrm{GeV}$. For full information please see the conference paper [9].

Table 1. The basic event selection of the three channels [9].

\begin{tabular}{|c|c|c|}
\hline 0-lepton & 1-lepton & 2-lepton \\
\hline 0 loose & $\begin{array}{c}1 \text { tight } \\
+0 \text { loose }\end{array}$ & $\begin{array}{l}1 \text { medium } \\
+1 \text { loose }\end{array}$ \\
\hline $\begin{array}{c}2 b \text {-tags } \\
p_{\mathrm{T}}^{1}>45 \mathrm{GeV} \\
p_{\mathrm{T}}^{2}>20 \mathrm{GeV} \\
+\leq 1 \text { extra jets }\end{array}$ & $\begin{array}{c}2 b \text {-tags } \\
p_{\mathrm{T}}^{1}>45 \mathrm{GeV} \\
p_{\mathrm{T}}^{2}>20 \mathrm{GeV} \\
+0 \text { extra jets }\end{array}$ & $\begin{array}{c}2 b \text {-tags } \\
p_{\mathrm{T}}^{1}>45 \mathrm{GeV} \\
p_{\mathrm{T}}^{2}>20 \mathrm{GeV} \\
-\end{array}$ \\
\hline $\begin{array}{c}E_{\mathrm{T}}^{\text {miss }}>120 \mathrm{GeV} \\
p_{\mathrm{T}}^{\text {miss }}>30 \mathrm{GeV} \\
\Delta \phi\left(E_{\mathrm{T}}^{\text {miss }}, p_{\mathrm{T}}^{\text {miss }}\right)<\pi / 2 \\
\min \left[\Delta \phi\left(E_{\mathrm{T}}^{\text {miss }}, j \text { jet }\right)\right]>1.5 \\
\Delta \phi\left(E_{\mathrm{T}}^{\text {miss }}, b \bar{b}\right)>2.8\end{array}$ & - & $E_{\mathrm{T}}^{\mathrm{miss}}<60 \mathrm{GeV}$ \\
\hline- & $m_{\mathrm{T}}^{W}<120$ & $83<m_{\ell \ell}<99$ \\
\hline
\end{tabular}

Table 2. Further topological cuts for the three channels in separate $p_{\mathrm{T}}^{V}$ intervals [9].

\begin{tabular}{|c|c|c|c|c|}
\hline \multicolumn{5}{|c|}{ 0-lepton channel } \\
\hline$E_{\mathrm{T}}^{\text {miss }}(\mathrm{GeV})$ & $120-160$ & $160-$ & 200 & $>200$ \\
\hline$\Delta R(b, \bar{b})$ & $0.7-1.9$ & $0.7-$ & 1.7 & $<1.5$ \\
\hline \multicolumn{5}{|c|}{ 1-lepton channel } \\
\hline \multicolumn{5}{|c|}{\begin{tabular}{l|l|l|l|l|}
$0-50$ & $50-100$ & $100-150$ & 150 \\
\end{tabular}} \\
\hline$\Delta R(b, \bar{b})$ & \multicolumn{3}{|c|}{$>0.7$} & $<1.4$ \\
\hline$E_{\mathrm{T}}^{\mathrm{miss}}(\mathrm{GeV})$ & \multicolumn{3}{|c|}{$>25$} & $>50$ \\
\hline$m_{\mathrm{T}}^{W}(\mathrm{GeV})$ & \multicolumn{2}{|c|}{$>40$} & \multicolumn{2}{|l|}{ - } \\
\hline \multicolumn{5}{|c|}{ 2-lepton channel } \\
\hline$p_{\mathrm{T}}^{Z}(\mathrm{GeV})$ & \multirow{2}{*}{\multicolumn{2}{|c|}{\begin{tabular}{|c|c|c|}
$0-50$ & $50-100$ & $100-150$ \\
\multicolumn{2}{|c|}{$>0.7$}
\end{tabular}}} & $150-200$ & $>200$ \\
\hline$\Delta R(b, \bar{b})$ & & & $0.7-1.8$ & $<1.6$ \\
\hline
\end{tabular}

Events in the signal region are required to contain exactly two $b$-tagged jets, which are used to reconstruct the mass of the Higgs boson candidate, $m_{b \bar{b}}$. For 3-jet events the leading two jets are required to be $b$-tagged. The mass resolution is improved by adding the energy from muons within the jet to the total jet energy. A $p_{\mathrm{T}}$-dependent correction is also applied to account for biases in the response caused by resolution effects of the jets from the Higgs boson decay. The average resolution after these corrections is about $16 \%$.

\section{Background normalisation and control samples}

The dominant backgrounds are top, $W+$ jet and $Z+$ jet production, with $Z+$ jet production the largest for the 2-lepton channel, top and $W+$ jets largest for the 1-lepton channel, and all three making an approximately equal contribution to the 0-lepton channel.

Backgrounds are estimated using a combination of techniques based on the comparison of data and MC predictions. The shapes of most background distributions are taken from simulation with normalisation using control regions in data. The two exceptions are the multijet background, which is estimated using data, and the diboson background for which both the normalisation and shape are taken from simulation.

Control regions are used to obtain the normalisation of the top background from data in the 1- and 2-lepton channels. In the 1-lepton channel the control region is defined by requiring an additional jet, and for the 2-lepton channel by inverting the requirements on $m_{\ell \ell}$ and $E_{\mathrm{T}}^{\mathrm{miss}}$. Here top refers to the sum of $t \bar{t}$ and single top production, which are treated together.

Control distributions to study the $V+$ jets background are made by relaxing the requirement on $2 b$-tagged jets. Events are categorised according to the number of $b$ tagged jets to define the signal and control regions. The 0 -tag and 1-tag control regions contain exactly 0 or $1 b$ tagged jets. The pre-tag control region is the sum of the 0-tag, 1-tag control regions and 2-tag signal region. Studying these distributions using the high statistics of the $\sqrt{s}=8 \mathrm{TeV}$ dataset revealed limitations in the modelling of certain distributions by the Monte Carlo simulation particularly in terms of the vector boson $p_{\mathrm{T}}$ spectra. For a number of backgrounds the vector boson $p_{\mathrm{T}}$ distribution of the data falls more rapidly than predicted by the Monte Carlo. Therefore corrections were obtained from the high statistics pre-tag regions for both $W$ and $Z+$ jets.

The multijet background in each channel is estimated from data. It is found to be $\sim 1 \%$ in the 0-lepton channel using an 'ABCD method'. In this method, the data are divided into 4 regions based on the variables $\min \left[\Delta \phi\left(E_{\mathrm{T}}^{\text {miss }}\right.\right.$, jet $\left.)\right]$ and $\Delta \phi\left(E_{\mathrm{T}}^{\text {miss }}, p_{\mathrm{T}}^{\text {miss }}\right)$, such that three of the regions are dominated by background. The background in the signal region, defined by high $\min \left[\Delta \phi\left(E_{\mathrm{T}}^{\text {miss }}, \mathrm{jet}\right)\right]$ and low $\Delta \phi\left(E_{\mathrm{T}}^{\mathrm{miss}}, p_{\mathrm{T}}^{\text {miss }}\right)$ is then evaluated under the assumption that the variables are not correlated. In the 1-lepton channel a multijet template is obtained by reversing the track isolation requirements on the leptons and subtracting $W, Z$ and top contaminations. The multijet background in the 1-lepton channel ranges from $23 \%$ to $2 \%$ of the total background rapidly decreasing with $p_{\mathrm{T}}^{W}$. A shape template for the multijet background in the 
2-lepton channel is estimated from data by inverting the isolation and relaxing the lepton quality requirements. It is normalised to the signal selection using the $m_{\ell \ell}$ distribution. The multijet background is found to be $<1 \%$ for the 2-lepton channel.

The normalisations of the $V+c$ and $V+$ light backgrounds are determined by a maximum likelihood fit to control and signal regions in the 1- and 2-lepton channels. Events containing 0,1 and $2 b$-tagged jets and the top control regions of each channel are used. This flavour fit exploits the fact that the $b$-tagging has very different efficiencies for $b, c$ and light jets, such that the flavour composition varies significantly with the number of $b$ tagged jets. The normalisations of $Z+$ light, $Z+c, Z+b$, $W+$ light, $W+c, W+b$ and top are allowed to float in the fit. The distributions of the control and signal regions for one of the channels (1-lepton) after having been normalised by the result of the flavour fit is shown in Fig. 1 for the $\sqrt{s}=8 \mathrm{TeV}$ data. The flavour fit results for $V+c$ and $V+$ light jets are shown in Table 3 for both the $\sqrt{s}=7 \mathrm{TeV}$ and $\sqrt{s}=8 \mathrm{TeV}$ datasets as scale factors with respect to the predicted Monte Carlo cross sections. Consistent scale factors are obtained for both datasets except for $Z+c$ production. This is expected, because the two samples were generated using different programs, ALPGEN and SHERPA. Some of the $Z+c$ events arise from charm production in the parton shower of $Z+$ light parton events. However, in the ALPGEN samples used for the $\sqrt{s}=7 \mathrm{TeV}$ datasets, the limited statistics in the $Z+$ light sample meant that the contribution from charm from the shower was suppressed to improve the statistical precision from the MC. As a result, the larger scale factor from the flavour fit compensates for this missing charm component. Due to a possible signal contamination and the impact of systematics on the scale factors, the results of this fit are not used to determine the final top and $V+b$ background normalisations. These normalisations are determined later in the fit used to calculate the limits as described in section 5 .

Table 3. Rescaling factors obtained from a fit to the data for the $V+$ light and $c$-jet backgrounds [9]. The error includes statistical and systematic uncertainties.

\begin{tabular}{l|c|c}
\hline \hline & $\sqrt{s}=7 \mathrm{TeV}$ & $\sqrt{s}=8 \mathrm{TeV}$ \\
\hline$Z+c$ & $1.99 \pm 0.51$ & $0.71 \pm 0.23$ \\
\hline$Z+$ light & $0.91 \pm 0.12$ & $0.98 \pm 0.11$ \\
\hline$W+c$ & $1.04 \pm 0.23$ & $1.04 \pm 0.24$ \\
\hline$W+$ light & $1.03 \pm 0.08$ & $1.01 \pm 0.14$ \\
\hline \hline
\end{tabular}

\section{Results}

The statistical analysis has been carried out in different categories depending on the number of leptons, number of jets and the transverse momentum of the vector boson candidate. A binned likelihood function $\mathcal{L}(\mu, \theta)$ is constructed as the product of Poisson probability terms for each category. A signal strength parameter, $\mu$, multiplies the ex-

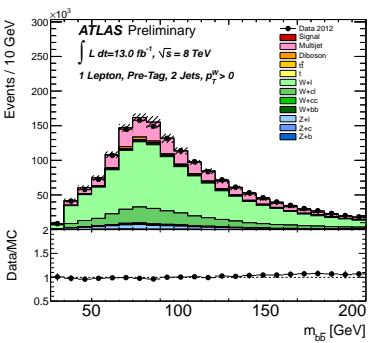

(a) 1-lepton, pre-tag

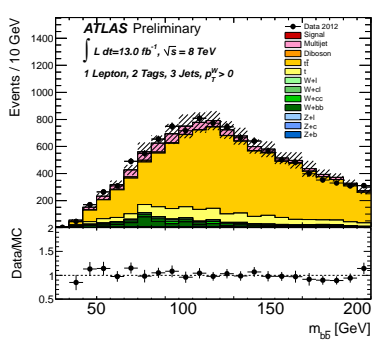

(c) 1-lepton, 2-tag, 3-jets

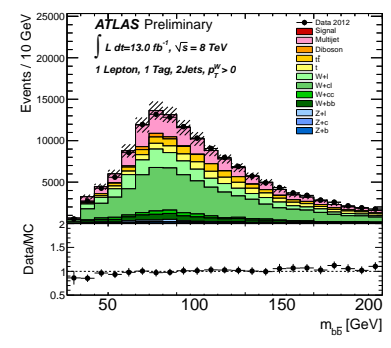

(b) 1-lepton, 1-tag

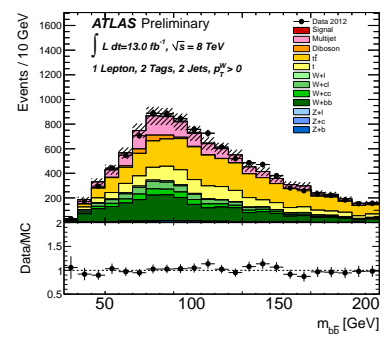

(d) 1-lepton, 2-tag
Figure 1. The $m_{b \bar{b}}$ distributions for the signal (bottom right) and control (top and bottom left) regions for the 1-lepton selection integrated over the bins of $p_{\mathrm{T}}^{V}$ [9]. The error bands indicate the size of the combined statistical and systematic uncertainty before the profile likelihood fit. See text for details of the definition of the control regions.

pected Standard Model Higgs boson production cross section in each bin. The dependence of the signal and background predictions on the systematic uncertainties is described by nuisance parameters, $\theta$, which are parametrised by Gaussian or log-normal priors. The expected number of signal and background events in each bin are functions of $\theta$. The parametrisation is chosen such that the rates in each category are log-normally distributed for a normally distributed $\theta$. The test statistic $q_{\mu}$ is then constructed according to the profile likelihood: $q_{\mu}=2 \ln \left(\mathcal{L}\left(\mu, \hat{\theta_{\mu}}\right) / \mathcal{L}(\hat{\mu}, \hat{\theta})\right)$, where $\hat{\mu}$ and $\hat{\theta}$ are the parameters that maximise the likelihood (with the constraint $0 \leq \hat{\mu} \leq \mu$ ), and $\hat{\theta}_{\mu}$ are the nuisance parameter values that maximise the likelihood for a given $\mu$. This test statistic is used to measure the compatibility of the background only model with the observed data and for exclusion intervals derived with the $C L_{\mathrm{s}}$ method [13, 14]. The normalisation of the top, $Z+b$ and $W+b$ backgrounds are allowed to float freely in the fit. The other backgrounds are constrained within their errors as described in section 4 . The resulting scale factors from the fit are shown in table 4 for both $\sqrt{s}=7 \mathrm{TeV}$ and $\sqrt{s}=8 \mathrm{TeV}$.

Diboson production with a $Z$ boson decaying to a pair of $b$-quarks and produced with either a $W$ or $Z$ boson has a very similar signature, but with a lower $p_{\mathrm{T}}^{Z}$ spectrum, $m_{b \bar{b}}$ peaking at lower values, and with a cross section $\sim 5$ times larger than Higgs boson associated production. Therefore a separate fit was made as a validation of the analysis procedure. This fit is performed as described above, except that there is no separation in $p_{\mathrm{T}}^{V}$ bins. In the fit the nor- 
Table 4. Rescaling factors obtained from the fit to the data for the $V+b$ and top backgrounds [9]. The error includes statistical and systematic uncertainties.

\begin{tabular}{l|l|l}
\hline \hline & $\sqrt{s}=7 \mathrm{TeV}$ & $\sqrt{s}=8 \mathrm{TeV}$ \\
\hline Top & $1.10 \pm 0.14$ & $1.29 \pm 0.16$ \\
$Z+b$ & $1.22 \pm 0.20$ & $1.11 \pm 0.15$ \\
$W+b$ & $1.19 \pm 0.23$ & $0.79 \pm 0.20$ \\
\hline
\end{tabular}

malisation of the diboson contributions is allowed to vary with a multiplicative scale, $\mu_{\mathrm{D}}$, with respect to the Standard Model expectation. A Standard Model Higgs boson with $m_{H}=125 \mathrm{GeV}$ is included as a background.

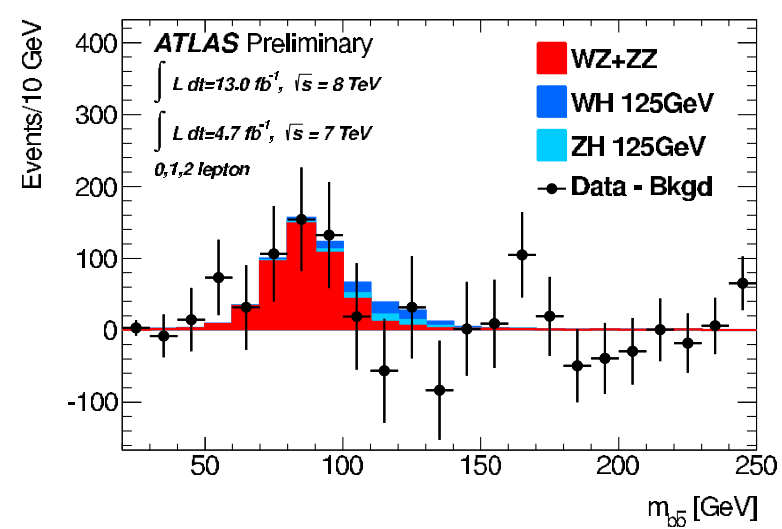

Figure 2. The $m_{b \bar{b}}$ distribution in data after subtraction of all backgrounds except diboson processes including SM Higgs boson from $W H$ and $Z H$ associated production [9]. The MC backgrounds are normalised according to the results of the global fit. Only statistical uncertainties are shown.

Figure 2 shows the distribution in data after subtracting all backgrounds except the diboson contributions. The backgrounds are evaluated after the results of the profile likelihood fit. An excess in the data compared to the background is observed and this excess is located at the expected mass for the vector boson signal. After combining the three channels and including all systematic uncertainties we measure $\mu_{\mathrm{D}}=1.09 \pm 0.20$ (stat.) \pm 0.22 (syst.). This corresponds to a significance of $4.0 \sigma$ and agrees with the Standard Model expectation of $\mu_{\mathrm{D}}=1$.

The profile likelihood fit with the Higgs boson signal strength floating is then performed with the diboson contribution fixed to its Standard Model expectation, constrained within an uncertainty of $11 \%$, as discussed above.

Figure 3 shows the 1-lepton $m_{b \bar{b}}$ distribution in $\sqrt{s}=$ $8 \mathrm{TeV}$ data after all selection criteria have been applied. The dashed bands indicate the size of the combination of the statistical and systematic uncertainties on the background processes after the likelihood fit. Agreement between data and background is observed within the systematic uncertainties.

Figure 4 shows the results for the $95 \%$ confidence level (CL) exclusion limits on the Higgs boson production cross

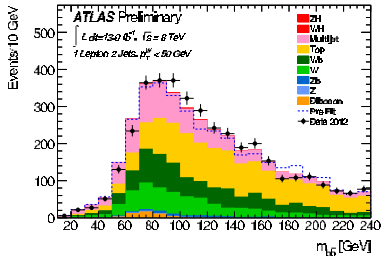

(a) $p_{\mathrm{T}}^{W}<50 \mathrm{GeV}$

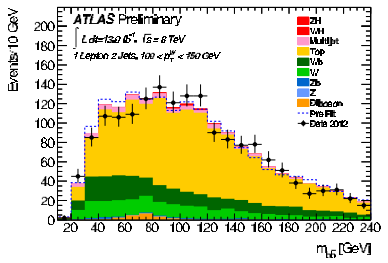

(c) $100<p_{\mathrm{T}}^{W}<150 \mathrm{GeV}$

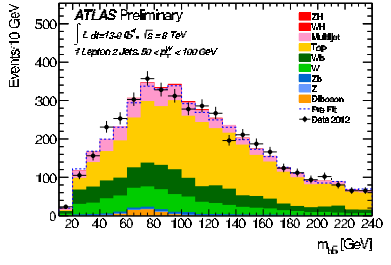

(b) $50<p_{\mathrm{T}}^{W}<100 \mathrm{GeV}$

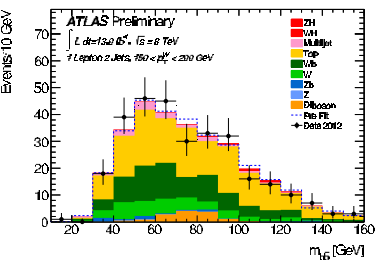

(d) $150<p_{\mathrm{T}}^{W}<200 \mathrm{GeV}$

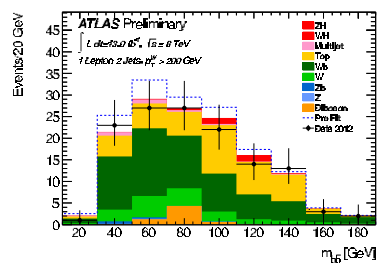

(e) $p_{\mathrm{T}}^{W}>200 \mathrm{GeV}$

Figure 3. The $m_{b \bar{b}}$ distribution in data for the five categories of the one lepton analysis [9]. The signal shown is for $m_{H}=$ $125 \mathrm{GeV}$. The background from the $V+$ jet process is separated into the $V+b$ and the sum of the $V+c$ and $V+$ light components. The background expectation is shown after the profile likelihood fit (solid) and compared to the predictions from the pre-fit Monte Carlo simulation (dashed). The size of the combined statistical and systematic uncertainty is indicated by the hashed band.

section in the mass range $110-130 \mathrm{GeV}$. The expected limits range from 1.5 to 3 times the SM expectation over the full mass range for the $\sqrt{s}=8 \mathrm{TeV}$ dataset alone. The expected limits for $m_{H}=125 \mathrm{GeV}$ are 3.3 and 2.5 times the Standard Model expectations for $\sqrt{s}=7 \mathrm{TeV}$ and $\sqrt{s}=8 \mathrm{TeV}$, respectively. For the $\sqrt{s}=7 \mathrm{TeV}$ data, this presents a significant improvement compared to the expected sensitivity of 4.0 times the Standard Model expectation reported in Ref. [10]. No significant excess is observed. The corresponding observed limits are 1.8 and 3.4 times the Standard Model expectations. This corresponds to a deficit of data events compared to the expectation from background processes of almost $2 \sigma$ in the $\sqrt{s}=7 \mathrm{TeV}$ dataset and an excess of about $1 \sigma$ in the $\sqrt{s}=8 \mathrm{TeV}$ dataset. The observed limit for the combination of the $\sqrt{s}=7 \mathrm{TeV}$ and $\sqrt{s}=8 \mathrm{TeV}$ datasets is 1.8 times the Standard Model expectation with an expected limit of 1.9 times the Standard Model. A Standard Model Higgs boson with $m_{H}=110 \mathrm{GeV}$ is excluded at the 95\% CL.

Figure 5 shows the expected and observed $p_{0}$ value in the same mass range. The quantity $p_{0}$ represents the probability that a background-only experiment would yield a result that is more signal-like than the observed result. 


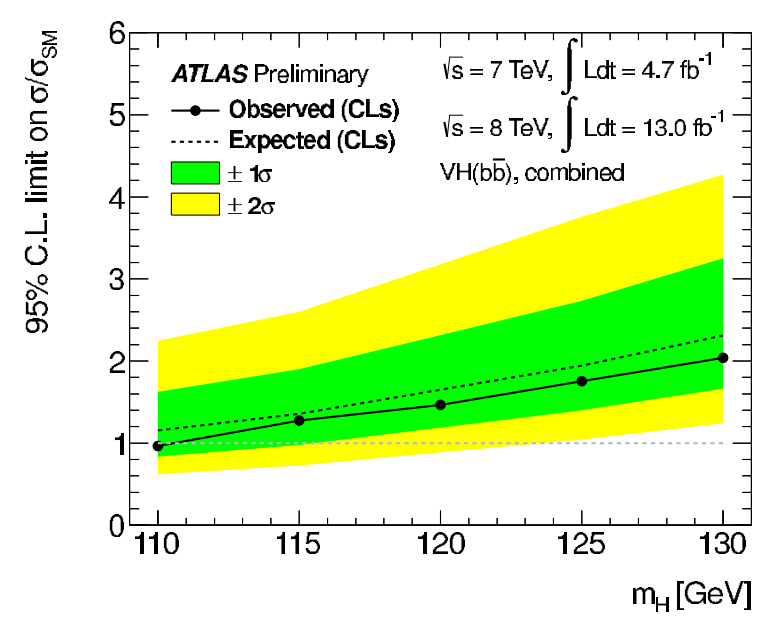

Figure 4. Expected (dashed) and observed (solid) $C L_{\mathrm{s}}$ limit on the normalised signal strength as a function of $m_{\mathrm{H}}$ for all channels [9].

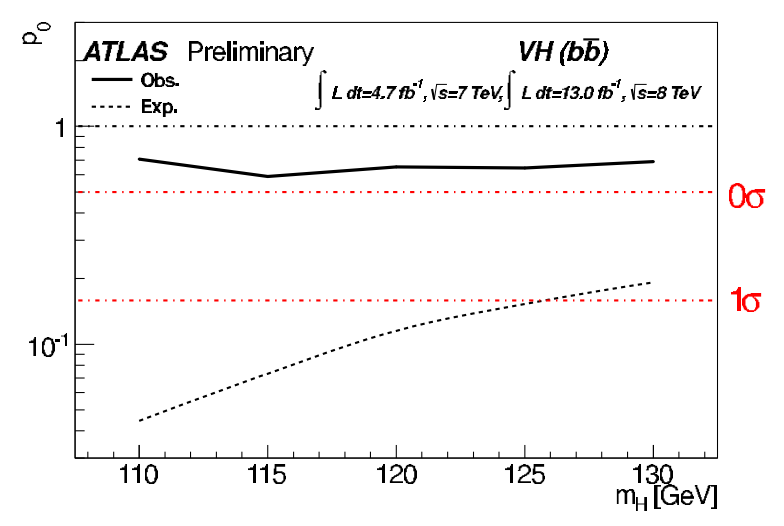

Figure 5. The observed probability, $p_{0}$, in the background-only scenario (solid line) as a function of $m_{\mathrm{H}}$ [9]. The dashed line shows the corresponding expectation for the signal+background hypothesis at the given value of $m_{\mathrm{H}}$.

For $m_{H}=125 \mathrm{GeV}$, the observed $p_{0}$ values are 0.97 and 0.17 for the $\sqrt{s}=7 \mathrm{TeV}$ and $\sqrt{s}=8 \mathrm{TeV}$ datasets, respectively. The expected $p_{0}$ values in the presence of a Standard Model Higgs boson are 0.26 and 0.20, respectively. The combination of both datasets yields an observed (expected) $p_{0}$ value of $0.64(0.15)$. We measure $\mu=$ $-2.7 \pm 1.1$ (stat.) \pm 1.1 (syst.), $\mu=1.0 \pm 0.9$ (stat.) \pm 1.1 (syst.) and $\mu=-0.4 \pm 0.7$ (stat.) \pm 0.8 (syst.) in the $\sqrt{s}=7 \mathrm{TeV}$, $\sqrt{s}=8 \mathrm{TeV}$ and combined datasets.

\section{Summary}

We have presented the search by the ATLAS experiment for the Standard Model Higgs boson produced in asso- ciation with a $W$ or $Z$ boson, where the decay channels $Z H \rightarrow v \bar{v} b \bar{b}, W H \rightarrow \ell v b \bar{b}$ and $Z H \rightarrow \ell^{+} \ell^{-} b \bar{b}$ have been considered. The analyses have been carried out in different categories depending on the number of leptons, number of jets and the transverse momentum of the vector boson candidate. No significant excess is observed. For $m_{H}=125 \mathrm{GeV}$, the observed (expected) upper limit on the production cross section times the branching ratio is found to be 1.8 (1.9) times the Standard Model prediction for the combined $\sqrt{s}=7 \mathrm{TeV}$ and $\sqrt{s}=8 \mathrm{TeV}$ datasets. observed (expected) upper 95\% CL limit on the cross section times the branching ratio is 1.8 (1.9) times the Standard Model prediction. A Standard Model Higgs boson with $m_{H}=110 \mathrm{GeV}$ is excluded. As a validation of the analysis method, the production of diboson pairs, $W Z$ and $Z Z$, with a $Z$ boson decaying to $b \bar{b}$, has been observed with a significance of 4.0 standard deviations and a rate compatible with the Standard Model expectation. The latest $t \bar{t} H$ measurement, with the Higgs boson decaying into two bottom quarks $\left(H \rightarrow b \bar{b}\right.$, used $4.7 \mathrm{fb}^{-1}$ of LHC data at $\sqrt{s}=7 \mathrm{TeV}$ and focused on the semileptonic decay mode of the $t \bar{t}$ system. For a SM Higgs boson with $m_{H}=125 \mathrm{GeV}$, the observed (expected) 95\% CL upper limits are 13.1 (10.5) times the Standard Model using $t \bar{t} H$.

For full information on both analyses please see the conference papers $[9,11]$.

\section{References}

[1] F. Englert, R. Brout, Phys. Rev. Lett. 13, 321 (1964)

[2] P.W. Higgs, Phys. Rev. Lett. 13, 508 (1964)

[3] P.W. Higgs, Phys. Lett. 12, 132 (1964)

[4] G. Guralnik, C. Hagen, T. Kibble, Phys. Rev. Lett. 13, 585 (1964)

[5] ATLAS collaboration, Phys.Lett. B716, 1 (2012), 1207.7214

[6] CMS collaboration (CMS), Phys.Lett. B716, 30 (2012), 1207.7235

[7] A. Djouadi, J. Kalinowski, M. Spira, Comput. Phys. Commun. 108, 56 (1998)

[8] T. Aaltonen et al. (CDF Collaboration, D0 Collaboration), Phys.Rev.Lett. 109, 071804 (2012), 1207.6436

[9] ATLAS collaboration, ATLAS-CONF-2012-161 (2012)

[10] ATLAS collaboration (ATLAS) (2012), 1207.0210

[11] ATLAS collaboration, ATLAS-CONF-2012-135 (2012)

[12] ATLAS collaboration, JINST 3, S08003 (2008)

[13] A.L. Read, J.Phys. G28, 2693 (2002)

[14] G. Cowan et al., Eur. Phys. J. C71, 1554 (2011), 1007.1727 